Proceedings of the 2011 Winter Simulation Conference

S. Jain, R. R. Creasey, J. Himmelspach, K. P. White, and M. Fu, eds.

\title{
MODELING AND SIMULATION OF MILITARY TACTICAL LOGISTICS DISTRIBUTION
}

\author{
Samir Sebbah \\ Center for Operational Research \& Analysis \\ Defence R\&D Canada \\ 101 Colonel By Drive, Ottawa, ON \\ Canada K1A 0K2
}

\author{
Ahmed Ghanmi \\ Center for Operational Research \& Analysis \\ Defence R\&D Canada \\ 101 Colonel By Drive, Ottawa, ON \\ Canada K1A 0K2
}

\author{
Abdeslem Boukhtouta \\ Decision Support Systems \\ Defence R\&D Canada - Valcartier \\ 2459, boulevard Pie-XI, Nord Val-Bélair \\ Québec, Canada G3J 1X5
}

\begin{abstract}
The military tactical logistics planning problem addresses the issue of distributing heterogeneous commodities in a theater of operations using a combination of heterogeneous transportation assets such as logistics trucks and tactical helicopters. The Canadian Forces requires a decision support tool to examine the trade-off between the cost of the support and its effectiveness during sustainment operations. In this study, a mathematical optimization algorithm and a simulation module to build cost efficient and effective military tactical logistics are developed. Details of the optimization algorithm along with several example applications are presented to demonstrate the methodology. The simulation results are focused on the trade-off between cost and lead-time within which demands are required, and on the optimal fleet mix of transportation assets to respond to the different requirements of deployed forces.
\end{abstract}

\section{INTRODUCTION}

The military tactical logistics planning problem addresses the issue of distributing heterogeneous commodities (e.g., food, medical material, construction material, ammunition, etc.) from Main Operating Bases (MOBs) to Forward Operating Bases (FOBs) in a theater of operations using a combination of heterogeneous transportation assets such as logistics trucks and tactical helicopters. FOBs are the front-line bases used by deployed troops, while MOBs are the backward coordination bases. In the design of supply chain strategies, organizations struggle for efficiency and effectiveness, which are related to the cost to achieve an objective and the degree of satisfaction of that objective. In the military, the trade-off between efficiency and effectiveness appears at different levels during the deployment and sustainment phases. During the deployment phase, the operational support goals are to ensure the deployment speed while keeping its cost at its lowest value. Similarly, during the sustainment phase, the objective is to ensure that the deployed forces are able to achieve their objectives while minimizing the cost to provide the required support level.

Given the amount of supplies the Canadian Forces (CF) is moving during each mission and the increasing demand for effective support, it has become a primordial issue to optimize the sustainment cost in order to provide effective support for deployed forces. The main challenge in the design of military tactical logistics is to find the loading and routing of an optimal fleet mix of transportation assets taking into 
consideration the security of transported commodities and reliability of transportation assets. Furthermore, given that different demands are issued with different priorities, i.e., required within different lead-times, then, another challenge is to find the optimal way to supply all the deployed forces without affecting the support provided to low-priority demands and increasing the support cost.

In order to assist the $\mathrm{CF}$ in building effective and cost efficient sustainment strategies, we develop a tactical logistics support framework including a module for field and operational characteristics simulation and an optimization module based on a large scale tool namely Column Generation (CG). Our main objective is to develop loading and routing strategies of an optimal fleets of transportation assets, including trucks and helicopters, given the field and transportation assets characteristics.

Our military tactical logistics problem is $\mathscr{N} \mathscr{P}$-Hard since it generalizes the Capacitated Vehicle Routing Problem (CVRP) and the Bin Packing Problem (BPP). Several solution approaches to handle the loading and routing of transportation assets based on heuristics and exact algorithms have been proposed to optimize the operational cost and meet different loading and routing constraints. Toth and Vigo (2002) presented an exhaustive review of the several extensions proposed for the VRP and made a classification of the different extensions according to some characteristics: (i) topology: demand locations to be satisfied, their number, number of depots, etc.; (ii) vehicles: homogeneous/heterogeneous fleet, fixed or variable fleet size, similar or different capacities, time windows for vehicle availability; (iii) supply strategy: split delivery, mixed delivery, time windows; (iv) objective: minimize the supply footprint, penalties in case where the service requirements are not met, penalties implying fleet size. Some previous studies of the VRP have considered different versions of the vehicles loading problem. Iori et al. (2007) proposed an exact approach based on branch-and-cut for the vehicle routing problem with two-dimensional loading constraints. The considered case is a symmetric capacitated vehicle routing problem in which a fleet of $K$ identical vehicles are used to serve customers. All vehicles are identical and have a known lift capacity and a single rectangular loading surface. All items of a given customer must be assigned to a single vehicle (called item clustering constraints). Furthermore, sequential loading constraints are added: items of a customer must not be blocked by item of customers to be visited later along the route. Malapert et al. (2008) proposed a constrained programming based approach to the two-dimensional pickup and delivery routing problem with loading constraints. Therein, the set of items requested by each client can be loaded onto a single vehicle, though, split deliveries are not allowed. The objective was to find a partition of the clients into routes of a minimal total cost, such that, for each route there exists a feasible loading of the items onto the vehicle loading surface that satisfies the capacity constraints.

The tactical logistics planning problem considered in this study involves vehicles routing and loading. However, it is quite different from the classical VRP. Indeed, we have: transportation assets of two modes (land, air) with different functional characteristics (capacity, speed, range, operational cost, reliability); multiple classes of commodities; commodities-clustering constraints to forbid transportation of securitysensitive classes of commodities with others; field-appropriate constraints to allow or forbid access to some routes for some classes of transportation assets; transportation-security constraints to allow or forbid loading of some security-sensitive commodities on some transportation assets; and lead-time constraints. Based on these new practical aspects of the problem, the routing and loading problems have to be re-designed in order to meet the different requirements. To the best of our knowledge this problem has not been dealt with in the literature, and this study is the first to include the different practical aspects of the problem.

This article is organized as follows. Section 2 gives an overview of our tactical logistics network model. Section 3 provides mathematical optimization models and solution algorithms for the considered problem. Section 4 presents the experimental results and discusses the trade-off between cost and lead-time, and between cost and nature of routes. Section 5 concludes the article.

\section{TACTICAL LOGISTICS SUPPORT MODEL}

In this section, we present our tactical logistics support model. We introduce some functional characteristics of the candidate transportation assets, and give details about the cost model and the support network topology. 
These components and models are studied together as they are holding an important part in our design of tactical support strategies.

\subsection{Functional Characteristics of Transportation Assets}

In military tactical logistics, a variety of classes of trucks and helicopters are used to deliver commodities to deployed troops. Depending on the transportation requirements of the commodities and the nature of the terrain, some classes of trucks and helicopters may be preferred over others. This is mainly valid when, for example, the required class of commodities is ammunition which requires reliable transportation assets.

In our model, transportation assets of the same class have similar functional characteristics. Associated to each transportation asset are its cruising speed, range, operational cost, payload and bulk capacity, reliability, set of potential commodities it can transport given its reliability level, and types of routes it can travel on to guarantee a safe transportation. The payload and bulk capacities define the maximum weight and volume capacities of the transportation asset. The reliability of a transportation asset defines its ability to transport some security-sensitive commodities. Another functional characteristic of transportation assets considered in this study is their ability to travel on routes of the terrain where forces are deployed.

\subsection{Transportation Safety}

In order to provide safe transportation of commodities, some classes of commodities are never loaded and transported together on the same transportation asset. This case of figure happens more often with ammunition and dangerous commodities. In order to avoid these loading patterns, commodities-clustering constraints are defined. Though, according to these constraints commodities of the same class may be clustered together but never with other commodities of other classes.

\subsection{Network Support Topology}

Figure 1 shows an example of a tactical logistics theater lay-out.

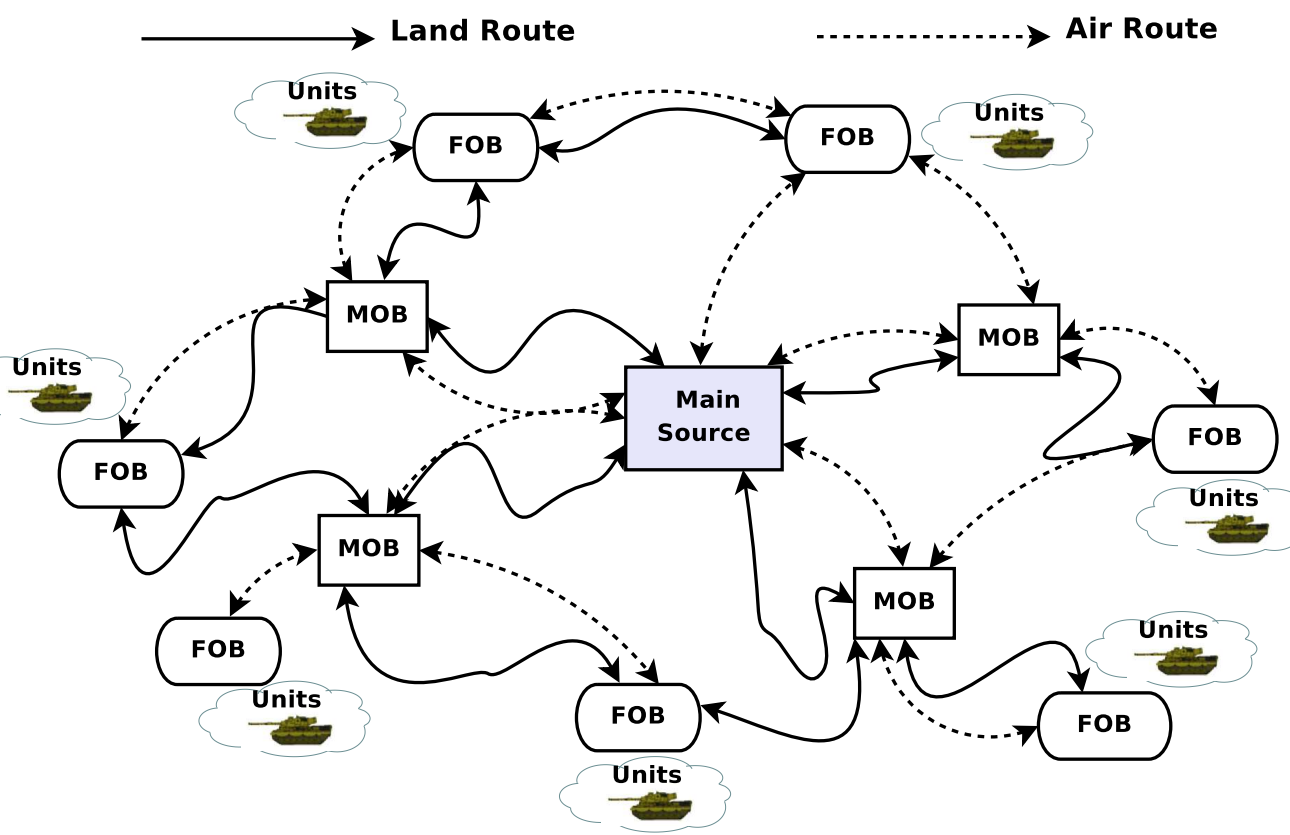

Figure 1: Tactical logistics network topology. 
In terms of supply flows, received material at the in-theater main source, which could be a depot or a $\mathrm{MOB}$, are delivered to FOBs through intermediate MOBs to meet the tactical requirements. Commodities are delivered in standard pallets of similar size but different weights. Our tactical logistics network topology is composed of a set of locations (MOBs, FOBs) interconnected by arrows which define the supply routes. Two network topologies, overlapping on some arrows and sharing all the locations, are shown to distinguish between land and air routes. We suppose given the land-routes, but the air routes are set up based on the reach-ability of the different locations by air and the range of the helicopters. Though, if any of the helicopters can reach point $B$ from point $A$, then a corresponding air route is added to illustrate that in the support network topology.

In our support model, demands for commodities issued at FOBs are required within different lead-times. We suppose and simulate different arrival rates of demands depending on the size of the deployed troops at the FOBs and the type of conducted missions (e.g., peacekeeping). Given the distances of routes and the ranges of transportation assets, refueling or transshipment stops may be required in order to deliver commodities from the main source to some FOBs. Indeed, in our network topology not all land and air routes can be used by transportation assets of all classes. If the range of a transportation asset is lower than the distance of a given route, then, the asset will not be used on that route. Though, if lead-time constraints have to be met, then, stops have to be pre-planned in the selection process of transportation assets to offset their negative effects. Furthermore, if the land-routes are of different nature, then, transshipment may be required at some intermediate locations in order to adapt to the new routes and provide safe and reliable transportation. These constraints are further explained in Section 3.

\subsection{Support Cost}

The support cost in our model is measured by the cost to operate the selected transportation assets. The cost model is set as a combination of different cost components (National Defence of Canada 2010), including the operating cost, crew cost, spare-part cost, amortized capital cost, etc. The operating cost of a transportation asset depends on its hourly cost rate, its cruising speed, and the length of the traveled distance (or time). Equation (1) gives the mathematical expression of the operating cost.

$$
\text { Operating cost }(\$)=\frac{\text { HourlyCost Rate }(\$ / \mathrm{h}) \times \text { Distance }(\mathrm{km})}{\text { Cruising Speed }(\mathrm{km} / \mathrm{h})}
$$

In order to optimize the selection process of transportation assets, the advantage will be given to transportation assets of classes, which offer a good ratio between their hourly cost and cruising speed to achieve the targeted tactical support.

\section{OPTIMIZATION MODELS}

In this section, we develop mathematical models to optimize the fleet-size and at the same time decide on the routing and loading of the selected transportation assets to meet the demands of the deployed forces. We adopt a CG decomposition approach and add Gomory-Chvátal rank-1 cutting planes (Chvátal 1973, Gomory 1960) to strengthen the linear formulation and derive integer solutions. CG is an efficient optimization method for solving large scale linear programs and its performance unfold in solving integer linear programming problems (Laporte 1992, Ribeiro and Soumis 1994). The main idea behind CG is that as most of the variables of the Integer Linear Program (ILP) model will not be part of the final optimal solution, then, considering only a subset of the most promising variables would be the only approach to reduce the size of the problem and increase the scalability of the solution method.

Our CG decomposition approach is based on the separation of the optimization of the tactical support plans and their design. A support plan $p \in \mathscr{P}$, is a combination of heterogeneous transportation assets distributed along the support network routes to transport different amounts of commodities to different 
destinations. The distribution is performed in a way that sends at most one transportation asset on each route. We define these sets and parameters:

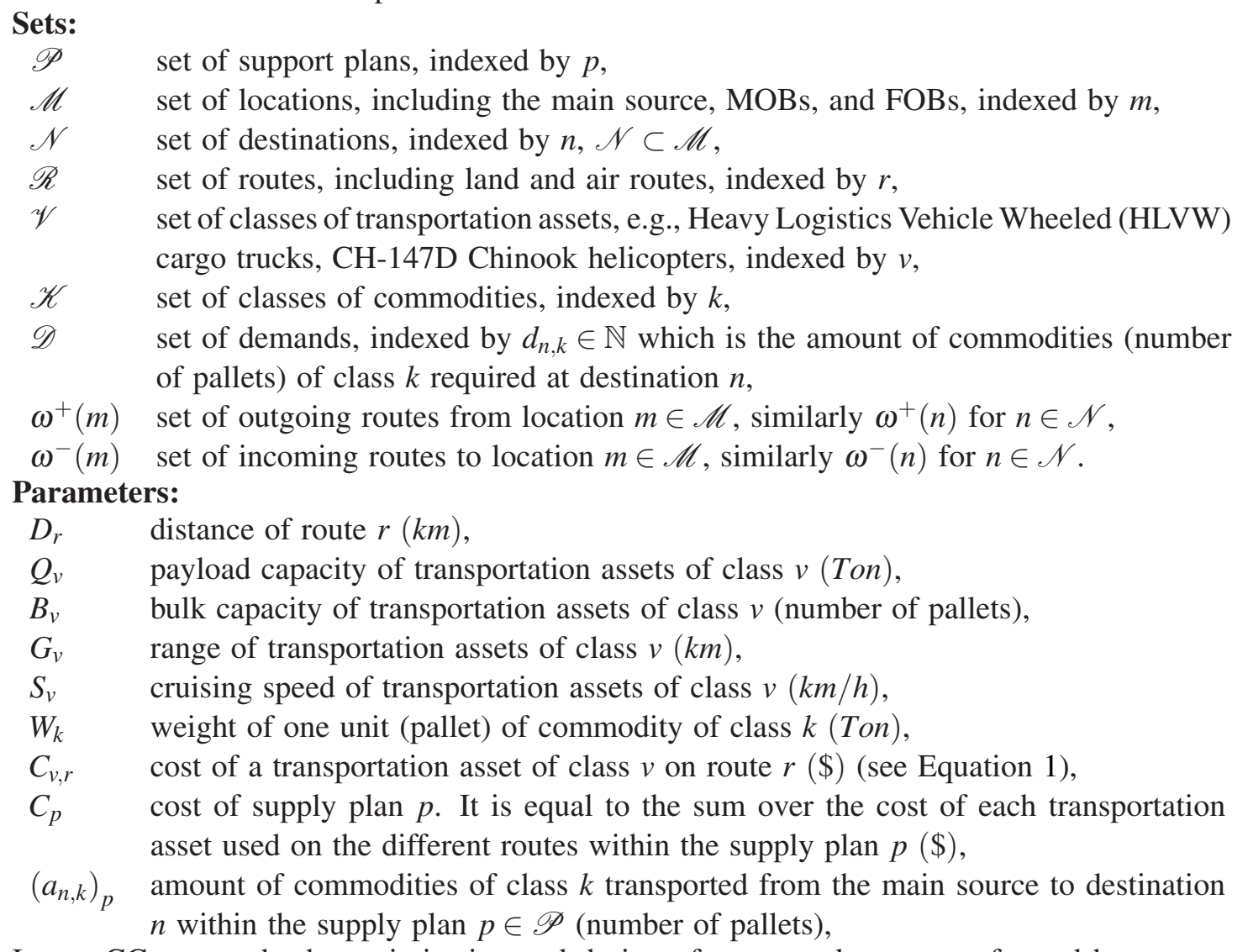

In our CG approach, the optimization and design of support plans are performed by two programs: the master and pricing programs, respectively. These two programs are presented below.

\subsection{Master Model}

In the master model, we optimize the selection of support plans $p \in \mathscr{P}$. We define $u_{p} \in \mathbb{N}$, for each plan $p \in \mathscr{P}$, as the number of identical copies of $p$. The master ILP model is given as follows:

Minimize:

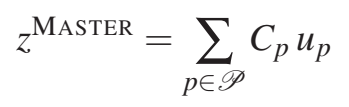

subject to:

$$
\begin{array}{ll}
\operatorname{la}_{p \in \mathscr{P}}\left(a_{n, k}\right)_{p} u_{p}=d_{k, n} & n \in \mathscr{N}, k \in \mathscr{K} \\
u_{p} \in \mathbb{N} & p \in \mathscr{P} .
\end{array}
$$

The objective function measures the cost of the candidate support plans $p \in \mathscr{P}$. Constraints (2) are the demand constraints to ensure that the requested commodities (number of pallets) of each class $k \in \mathscr{K}$ by each destination $n \in \mathscr{N}$ are met. Constraints (3) define the domain of variables $u_{p}$. Recall that the restricted master model, we used in the CG framework, is a restricted version of the relaxation of the above model obtained by replacing $u_{p} \in \mathbb{N}$ in Constraints (3) by $u_{p} \in \mathbb{R}_{+}$for all $p \in \mathscr{P}$. 


\subsection{Pricing Model}

The pricing problem, which is used to generate a promising support plan when it is called, corresponds to the minimization of the reduced cost of the restricted master problem subject to a set of supply plan design constraints. In our optimization model, we do not assume any specific number of transportation assets, rather, the only available input information is the classes of transportation assets. We define the following variables and parameters of the pricing problem for designing a single support plan:

Variables

$$
x_{v, r}= \begin{cases}1 & \text { if a transportation asset of class } v \text { is used on route } r \\ 0 & \text { otherwise }\end{cases}
$$

$y_{n, k} \in \mathbb{N}$ for each destination $n \in \mathscr{N}$ and class of commodities $k \in \mathscr{K}$, it is equal to the amount of commodities $k$ transported to destination $n$.

$y_{n, k, r} \in \mathbb{N}$ for each destination $n \in \mathscr{N}$, commodity $k \in \mathscr{K}$, and route $r \in \mathscr{R}$, it is equal to the amount of commodities of class $k$ transported to destination $n$ along route $r$.

Parameters

$$
v, k= \begin{cases}1 & \text { if transportation assets of class } v \text { can transport commodities of class } k \\ 0 & \text { otherwise }\end{cases}
$$

\section{The ILP Pricing Model:}

Minimize:

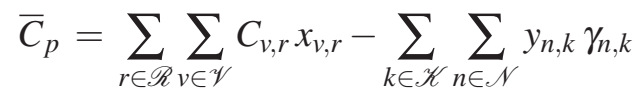

where $n, k$ are the values of the dual variables associated with constraints (2). subject to:

- Flow conservation constraints (4): they are used to route flows of commodities from the main source to the destinations (FOBs).

$$
r_{r \in+(m)} y_{n, k, r}-y_{r \in-(m)} y_{n, k, r}=\left\{\begin{array}{cl}
y_{n, k} & \text { if } m \text { is the main-source } \\
-y_{n, k} & \text { if } m \in F O B s \\
0 & \text { otherwise }
\end{array} \quad k \in \mathscr{K}, n \in \mathscr{N}\right.
$$

- Support plan and land specific constraints (5 and 6): Constraints 5 are used for each route $r \in \mathscr{R}$ to set the maximum number of transportation assets of all used classes to at most one. In this selection, only transportation assets $v$ with a range greater or equal to the distance of the selected route $r$ are allowed. Constraints 6 set the access rights to travel on routes for transportation assets of classes by fixing variables $x_{v, r}$.

$$
\begin{aligned}
& x_{v, r} \leq 1 \quad r \in \mathscr{R} \\
& \begin{array}{c}
\nu \in \mathscr{V} \\
G_{v} \leq D_{r}
\end{array} \\
& x_{v, r} \leq{ }_{v, r} \quad r \in \mathscr{R}, v \in \mathscr{V}
\end{aligned}
$$

where

$$
v, r= \begin{cases}1 & \text { if route } r \text { can be used by transportation assets of class } v \\ 0 & \text { otherwise }\end{cases}
$$


- Capacity constraints (7 and 8): are payload and bulk transportation capacity constraints, respectively. These constraints are used to limit the total amount of transported pallets of all commodities by the payload or bulk capacity of the selected transportation asset.

$$
\begin{array}{rl}
{ }_{n \in \mathscr{N} k \in \mathscr{K}} W_{k} y_{n, k, r} \leq \underbrace{}_{v \in \mathscr{V}} Q_{v} x_{v, r} & r \in \mathscr{R} \\
y_{n \in \mathscr{N} k \in \mathscr{K}} y_{n, k, r} \leq{\underset{v \in \mathscr{V}}{ } B_{v} x_{v, r}} & r \in \mathscr{R}
\end{array}
$$

- Lead-time and commodities-clustering constraints: these categories of constraints are also considered in our model. The lead-time constraints are used to set up an upper bound on the transportation and transshipment/refueling times to get commodities from the source to their destination. The commodities-clustering are added by forbiding some clustering patterns of commodities.

The output of the pricing problem is a support plan. Within a support plan, on each route $r \in \mathscr{R}$ it is scheduled at most one vehicle (truck or helicopter) of a given class $v \in \mathscr{V}$ carrying a set of commodities $k \in \mathscr{K}$ to a set of destinations $n \in \mathscr{N}$. The flow of commodities of class $k \in \mathscr{K}$ that reaches a destination $n$ is given by variable $y_{n, k}$, and on each route $r$ by variables $y_{n, k, r}$.

\subsection{Solution Algorithm}

The flowchart in Figure 2 illustrates our CG based algorithm to the tactical logistics problem. The CG solution algorithm consists of executing the pair master-pricing problems until no improving variable (support plan) could be found. Each time a promising support plan (improves the value of the objective) is found it is added to the master problem, and the process re-starts again. If no improving plan is available, i.e., the reduced cost of the pricing problem is greater or equal to zero, then, a Gomory-Chvátal rank-1 separation problem is initiated. The separation problem will find, if any, a cutting plane to cut-off the fractional solution or return a certificate that no such cut is available. In case a cutting plane is found, it is added to the master problem, and the whole process re-starts again, otherwise, the process ends and a classical branch and bound algorithm is started to derive an integer solution. During the branch and bound algorithm the integer solution is derived using only the so far generated columns and cuts.

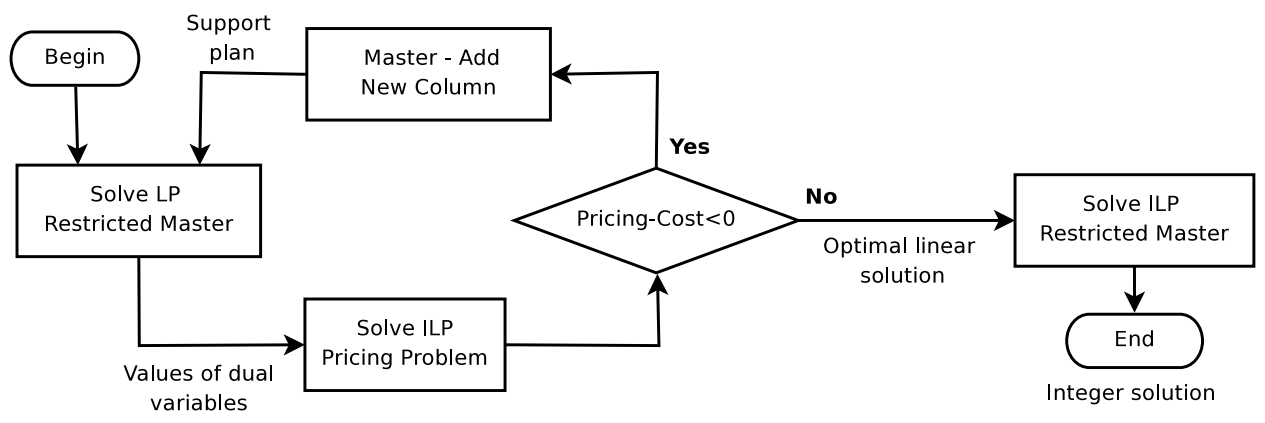

Figure 2: The solution algorithm.

\section{SIMULATION AND OPTIMIZATION RESULTS}

This section presents our simulation and optimization results obtained when solving the tactical logistics problem using the proposed algorithm. The integer solutions are obtained within an integrality gap less than $2 \%$ in all performed experiments. 


\subsection{Data Inputs}

We are given a logistics network topology of 7 nodes and 24 routes composed as follows: 1 main source, 3 MOBs, 3 FOBs, 12 land-routes, and 12 air routes. Recall that the air routes are established based on the reach-ability of the different locations.

From a transportation requirement point of view, our classes of commodities are classified into three categories: general (e.g., food, clothing, personal demands, medical material), refrigerated (e.g., fresh food, rations, medical material), and ammunition (chemical and special weapons, bombs, explosives, mines, fuses, detonators, and others), referred to from now on as $K_{1}, K_{2}$, and $K_{3}$, respectively. In this study, we suppose that commodities are packed into pallets (standard transportation unit) of similar size. The average weights of the pallets depend on their contained class of commodities and described as follows: refrigerated 1 Ton/pallet, general 1.5 Ton/pallet, and ammunition 2 Ton/pallet .

Table 1 presents the characteristics of the different classes of transportation assets used in this study. As illustrated, two classes of helicopters: $\mathrm{CH}-147 \mathrm{D}$ Chinook and CH-146 Griffon, and three classes of trucks: LSVW (Light Support Vehicle Wheeled), MLVW (Medium Logistic Vehicle Wheeled), and HLVW (Heavy Logistic Vehicle Wheeled), are used.

In addition to their physical (payload and bulk capacities) and operational (hourly cost, range, and cruising-speed) characteristics, each transportation class is characterized by the set of classes of commodities that it can transport as described in the last column. This last characteristic is elaborated based on the reliability of the transportation classes and the sensitivity of the classes of commodities. Regarding the commodities clustering constraints, we forbid clustering of ammunitions with any of the two other classes.

Table 1: Characteristics of classes of transportation assets.

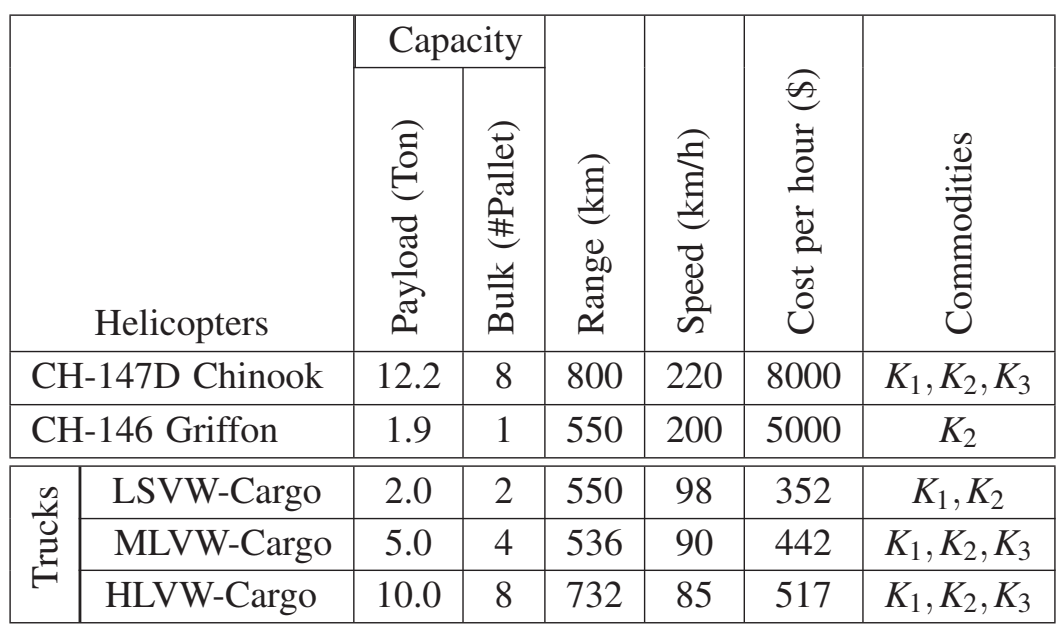

\subsection{Simulation Scenarios}

In order to illustrate our design methodology, three scenarios where demands for some commodities of a given class are higher compared to the two other classes, are considered. The rationale of this selection is that the needed amount of commodities of a given class depends on the supported missions, e.g., peacekeeping missions need more $K_{2}$, fighting missions need more $K_{3}$. Following this approach, each scenario is characterized by three arrival rates per week $i(i=1,2,3)$ of demands for commodities of classes $K_{i}(i=1,2,3)$. In the first scenario, the arrival rates of demands for the three classes of commodities are $1 \in[20,30], \quad 2$ and $3 \in[10,20]$. In the second and third scenarios, higher arrival rates of demands for commodities of classes $K_{2}$ and $K_{3}$ are received at the main source, respectively. The arrival rates in the second scenario are as follow: $2 \in[20,30], 1_{1}$ and ${ }_{3} \in[10,20]$, while in the third scenario they are $3 \in[20,30], \quad 1$ and ${ }_{2} \in[10,20]$. 


\subsection{Trade-off Between Lead-time and Support Cost}

In this section, we study the trade-off between support cost and lead-time. For each destination, we define the duration of the shortest transportation-time path (travel on routes plus servicing at intermediate locations) as $T_{S P}$.

Figure 3 presents the variation of the weekly support cost (y-axis) as a function of the normalized lead-time (x-axis) for the three different scenarios. The normalized lead-time values are obtained by dividing the different values by $T_{S P}$. Therein, $10 \%, 50 \%$, and $100 \%$ of commodities ( ) in each of the three scenarios are required within the illustrated normalized lead-time values on x-axis. From the three scenarios, firstly, we see that as the lead-time increases (relaxed lead-time) the support cost decreases. The lowest values of cost corresponds to the lead-time values $\geq 2.8$, and the highest to the lead-time values $\leq 1$ (i.e., $T_{S P}$ ). This holds in the three sub-figures with different values of .

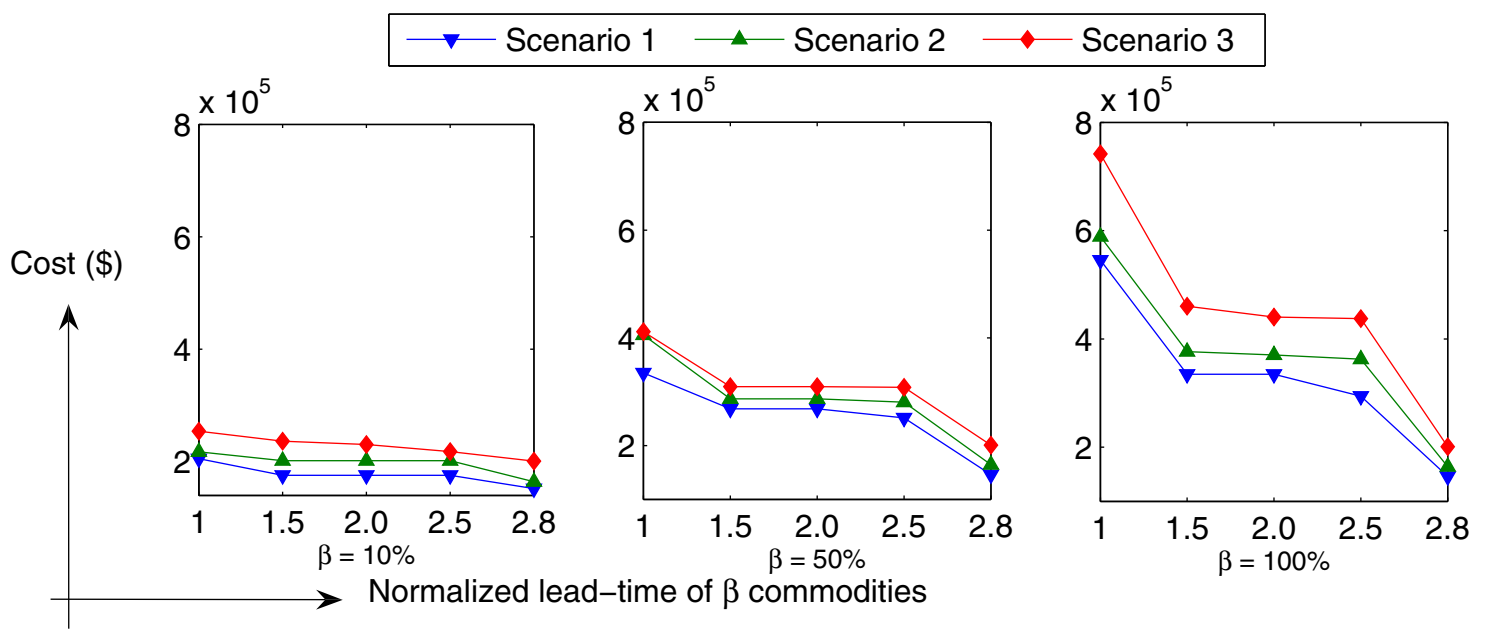

Figure 3: Trade-off between support cost and lead-time.

The difference in cost between two values of lead-time in the three scenarios depends on the percentage of commodities which are required within the two values of lead-time. The difference increases as the percentage of commodities required within the specified lead-time increases. Secondly, the highest support costs are recorded in the third scenario followed by the second scenario, and the lowest costs are recorded in the first scenario for all lead-time values. The main reason is due to the demand patterns in the three scenarios which require more or less of pallets of different weights.

Figure 4 presents the average number of lifts of the demands in the three scenarios performed by the selected transportation assets as a function of the percentage of commodities required within three selected values of lead-time $\left(T_{S P}, 2 T_{S P}\right.$, and $\left.2.8 T_{S P}\right)$. As reported, the totality of lifts are performed exclusively by $\mathrm{CH}-147 \mathrm{D}$ Chinook helicopters and HLVW trucks. In the first histogram of Figure 4, where the different percentages of commodities of the three distributions are required within a lead-time $\leq T_{S P}$, the number of lifts performed by $\mathrm{CH}-147 \mathrm{D}$ Chinooks increases as the percentage of commodities required within the selected lead-time (i.e., $T_{S P}$ ) increases. When all commodities $(100 \%)$ are required within a lead-time $\leq T_{S P}$ the totality of lifts are performed by CH-147D Chinooks. In the second histogram, where lead-time $\leq 2 T_{S P}$, the number of lifts performed by CH-147D Chinook helicopters and HLVW trucks are almost equal when $100 \%$ of commodities are required within the specified lead-time, and dominated by HLVW trucks for the two other percentages of commodities. In the last histogram, where lead-time $\leq 2.8$, the whole lifts are solely performed by HLVW trucks for the three lead-time values. For values of lead-time greater or equal to $2.8 T_{S P}$, the same pattern of lifts repeats itself. 


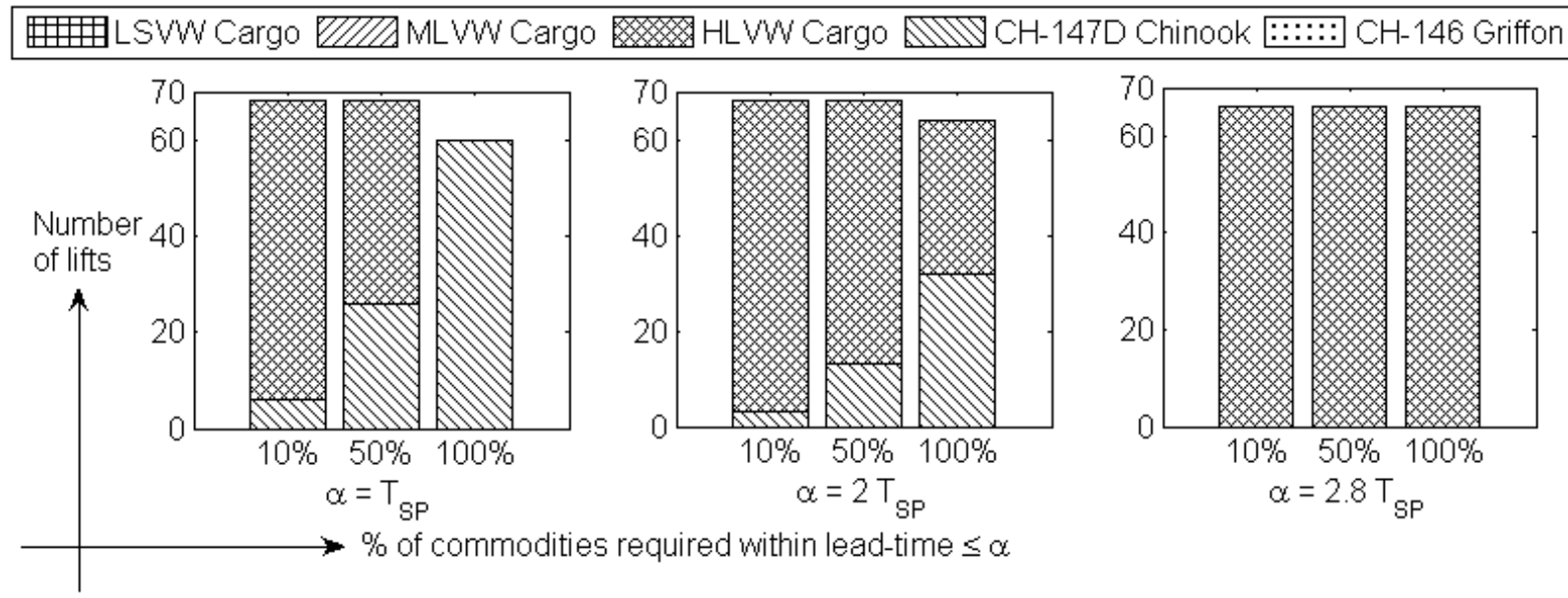

Figure 4: Number of lifts vs. lead-time.

\subsection{Impact of the Characteristics of Land-routes}

In this section, we study and analyze the effects of the type of routes on the support cost and the optimal fleet mix of transportation assets. We simulate different percentages of impractical land-routes by HLVW trucks (e.g., existence of limited capacity bridges). In this section, we relax the lead-time constraints, i.e., commodities could be delivered at any time.

Figure 5 presents the variation of the support cost (y-axis) as a function of the percentage of impractical land-routes by HLVW trucks (x-axis). Therein, it indicates that as the percentage of impractical routes by HLVW trucks increases the support cost increases. The support cost reaches its highest bound when the number of impractical routes is $\geq 50 \%$. Although there is still another $50 \%$ of practical routes by HLVW trucks, the support cost is the same as for $100 \%$ of impractical routes.

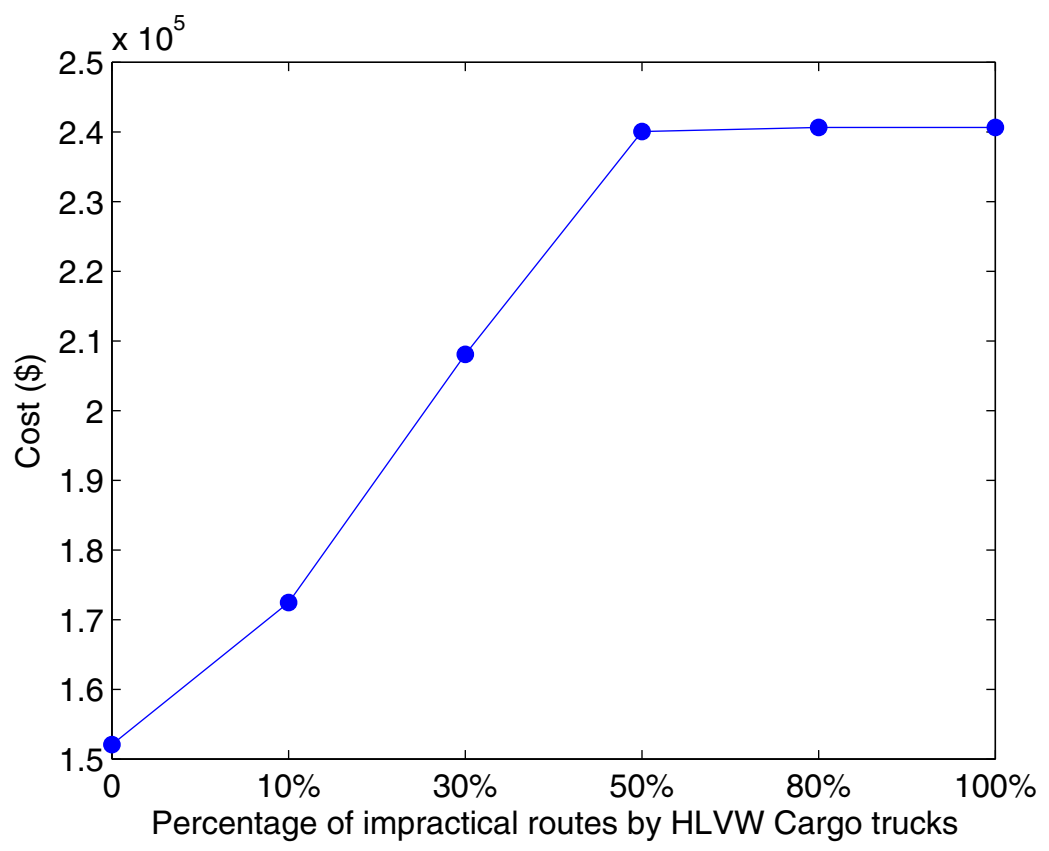

Figure 5: Trade-off between cost and percentage of impractical routes by HLVW trucks. 
Figure 6 presents the number of lifts realized by the transportation assets of the optimal fleet mix (y-axis) as a function of the percentage of impractical routes by HLVW trucks (x-axis). Therein, as the percentage of impractical routes by HLVW trucks increases, the total number of lifts increases (footprint increases), those realized by HLVW trucks decreases, those realized by MLVW trucks increases, and there is no lifts realized by CH-147D Chinooks. For $80 \%$ to $100 \%$ of impractical routes by HLVW trucks, all lifts are realized by MLVW trucks. The absence of lifts by CH-147D Chinooks is due to their operational cost and the relaxation of the lead-time constraint. Furthermore, as the number of impractical routes by HLVW trucks increases, the total available capacity per transportation asset decreases. Though, in order to keep up with the required transportation capacity, the number of lifts realized by the MLVW trucks increases because of their capacity.

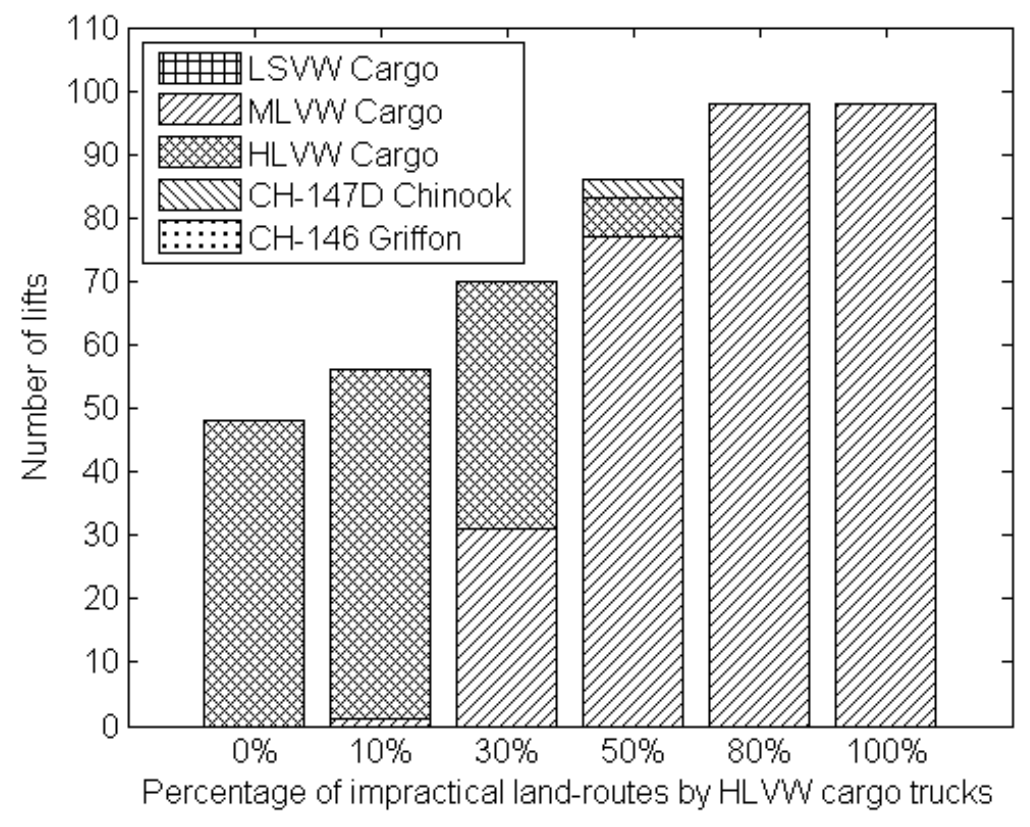

Figure 6: Number of lifts vs. impractical land-routes by HLVW trucks.

\section{CONCLUSION}

In this paper, we proposed a framework to support decisions in the design of military tactical logistics strategies. We developed an optimization method to minimize the operational cost, and a simulation framework to generate and simulate supply topologies and support assets with different operational characteristics. Our main focus was to study the trade-off between the support cost and a set of parameters including lead-time, safety of routes and commodities. We examined the variation of the support cost as a function of the percentage of commodities required within different lead-time values, and of the percentage of impractical routes by HLVW trucks. Furthermore, in order to get an insight into the compositions of the optimal fleets, we analyzed the number of lifts performed by five classes of transportation assets including two helicopter and three truck classes.

Our methodology, which is intended to be integrated into a logistics decision support system, can help decision makers to strike different balances between cost and the studied parameters. Different perspectives were explored and several alternatives were analyzed to help understand how to provide effective support without causing a high support cost escalation. 
Future research directions would be, firstly, to look very closely at how similar or different are the two problems of optimizing the tactical logistics footprints and the problem of optimizing the operational cost. Secondly, the problem of the convoy formation and escorting, and how it affects the selection of transportation assets and their routing. In this study, selected transportation assets are routed independently of each other. In convoy formation and routing, the speed of a transportation asset is defined by the speed of the convoy not its own cruising-speed. The convoy escorting, which is part of the operational cost, was not considered in this study. Including this parameter in the study will probably change the modeling and solution approaches and the optimal solution.

\section{REFERENCES}

Chvátal, V. 1973. "Edmonds polytopes and a hierarchy of combinatorial problems". Discrete Mathematics 4 (4): 305-337.

Gomory, R. 1960. “Solving linear programming problems in integers”. In Combinatorial Analysis, Symposia in Applied Mathematics X, 211-215. Providence, R.I.

Iori, M., J. González, and D. Vigo. 2007. "An exact approach for the vehicle routing problem with two-dimensional loading constraints". Transportation Science 41 (2): 253-264.

Laporte, G. 1992. "The vehicle routing problem: An overview of exact and approximate algorithms". European Journal of Operational Research 59 (3): 345-358.

Malapert, A., C. Guéret, N. Jussien, A. Langevin, and L.-M. Rousseau. 2008. "Two-dimensional Pickup and Delivery Routing Problem with Loading Constraints". Technical report, CIRRELT.

National Defence of Canada 2010. Cost Factors Manual. National Defence of Canada. Internal manual.

Ribeiro, C., and F. Soumis. 1994. "A Column Generation Approach to the Multi-Depot Vehicle Scheduling Problem". Operations Research 42:41-52.

Toth, P., and D. Vigo. 2002. The vehicle routing problem. Society for Industrial Mathematics.

\section{AUTHOR BIOGRAPHIES}

SAMIR SEBBAH is a visiting research fellow in the Centre for Operational Research and Analysis at the Defence R\&D Canada. He received a PhD degree in Electrical and Computer Engineering from the University of Concordia (Montreal, Qc, Canada) and a Master degree in Computer Science and Operational Research from the University of Paris 8. His research interests are in large scale optimization, modeling and simulation, and design and optimization of logistics strategies.

AHMED GHANMI received a B.Sc. degree in engineering, a Master degree and a Ph.D. in applied mathematics from the University Laval, Quebec, Canada. He is currently a senior defence scientist at the Defence Research and Development Canada - Centre for Operational Research and Analysis. His research interest includes military operations research, logistics and transportation modeling, decision analysis, simulation and optimization problems. Dr. Ghanmi has been leading different international research activities in NATO and technical cooperation programs.

ABDESLEM BOUKHTOUTA is a defence scientist with the Decision Support Systems Section of Defence Research and Development Canada - Valcartier, working in the field of information technology. He earned a Ph.D. degree from the decision system and operations department, Laval University, and holds a MS degree in Civil Engineering from Sherbrooke University, both from Quebec, Canada. His research interests include operations research and stochastic dynamic programming related fields, applied to defense logistics, planning, routing and scheduling problems. He is part of various national and international research and development programs involving universities and government organizations. Dr. Boukhtouta coauthored a book and many scientific publications. 